\title{
Dynamics of genital human papillomavirus (HPV) infections among males eventually being uncovered
}

\author{
Kari Syrjänen \\ Asian Journal of Andrology (2011) 13, 361-362; doi:10.1038/aja.2011.24; published online 18 April 2011
}

S tudies addressing the type-specific outcomes of cervical human papillomavirus (HPV) infections in women started emerging since the early 2000s, resulting in prolific literature and more profound understanding of the dynamic nature of these viral outcomes (incident infections, persistence and clearance) in the female genital tract. ${ }^{1,2}$ Despite the fact that scattered studies on genital HPV infections in males have been published since the late $1980 \mathrm{~s},{ }^{3}$ interest in these lesions has been meager, and studies published until now have failed to provide any information about the natural history of male genital HPV infections at the genotype level. ${ }^{4,5}$ During the past few years, however, HPV infections of the males have attracted tremendously increased attention, along with the introduction of prophylactic HPV vaccines for global use. ${ }^{6}$ Not unexpectedly, large prospective cohort studies, similar to those conducted for women since the early $1980 \mathrm{~s},{ }^{3,4}$ have been recently designed to cast further light on the natural history of male genital HPV infections as well. ${ }^{7,8}$ It is expected that these longitudinal studies will soon be mature enough to elaborate data equivalent to that already available on similar cohorts of women, i.e., start disclosing the outcomes of genital HPV infections at genotype-specific level also in the males.

A major step forward in this development represents the recent paper of Giuliano et al., which appeared in the Lancet on 28 February 2011. ${ }^{9}$ This study is among the first to provide genotype-specific information about the incidence and clearance times and rates of genital HPV infections among males, i.e., data that are potentially useful while designing cost-effectiveness models for male HPV

Department of Oncology and Radiotherapy, Turku University Hospital, Turku FIN-20521, Finland

Correspondence: Professor K Syrjänen (kari.syrjanen@ tyks.fi) vaccination worldwide. In addition, the authors performed a meticulous multivariate analysis to disclose the associated covariates (=determinants) of these viral events.

These novel data are derived from a prospective cohort study, known as HPV in men (HIM) cohort, which is an ongoing prospective study of the natural history of male HPV infections, conducted in three countries (Brazil, Mexico and the United States). ${ }^{9}$ The men were eligible to participate, most importantly, if (i) aged 18-70 years; (ii) were HIVnegative; (3) reported no history of penile or anal cancer; and (iv) also no previous diagnosis of genital or anal warts. All males were recruited from the general population, universities, or through organized healthcare systems. They were monitored at 6-month intervals for a median follow-up of 27.5 months (18.0-31.2), ${ }^{9}$ which is very similar to in several major women cohorts. ${ }^{1,2}$

Key reasons for the highly divergent HPV detection rates reported in the male genitalia in previous studies, ${ }^{3-5,7,8}$ obviously include the different sampling and HPV testing techniques. In the HIM cohort, particular attention has been paid to both of these. ${ }^{9}$ Thus, specimens were collected from the coronal sulcus, glans penis, shaft and scrotum, and all these were combined into one sample before DNA extraction. The authors have previously demonstrated the efficacy of this sampling strategy, resulting in highly reproducible detection of HPV DNA. HPV genotyping was done with the linear array method (Roche), which is a carefully validated test for this purpose, capable of detecting $37 \mathrm{HPV}$ genotypes.

Altogether, the authors enrolled 1159 eligible men in their study. ${ }^{9}$ New HPV infections were acquired at incidence rate of $38.4 / 1000$ person-months at risk (95\% CI: 34.3-43.0). Of the oncogenic HPV types, those with the highest incidence rate (per
1000 person-months at risk) included: HPV16, 51, 52 and 59, while the respective non-oncogenic HPV types were HPV6, 62, 84 and CP6108. Interestingly, these incidence rates are closely comparable to the accumulation of new genital HPV infections by women in a longitudinal setting. ${ }^{1,2}$

In multivariate (Cox) model, the authors could demonstrate that incident oncogenic HPV infections were significantly associated with (i) having a high number of lifetime female sexual partners $(\mathrm{HR}=2.4 ; 95 \% \mathrm{CI}$ : $1.38-4.18$, for 50 partners versus one partner); and (ii) number of male anal-sexual partners $(\mathrm{HR}=2.57$; 95\% CI: $1.46-4.49$, for three male partners versus no recent male partner). ${ }^{9}$ These observations are not unexpected or new (reported e.g., by us already in the early 1990s), ${ }^{4}$ and closely corroborate with the well established risk factors of incident HPV infections among women. ${ }^{1,2}$

As to the duration of HPV infections in these males, the times reported in the HIM cohort are once again practically identical to those reported in several major cohorts for women, as recently discussed. ${ }^{1,2}$ Accordingly, the median duration of HPV infection was 7.5 months (6.8-8.6 months) for any HPV, and 12.2 months (7.2-18.2 months) for HPV16 alone. In multivariate model, the authors examined the covariates of viral clearance. Importantly, the clearance of oncogenic HPV infection was shown to decrease in men who reported a high number of lifetime female partners $(\mathrm{HR}=0.49$; 95\% CI: $0.3-0.8$, for 50 partners versus one partner). Clearance was also delayed in males resident in Brazil and in Mexico, as compared with those in the United States. ${ }^{9}$ In the other way around, the number of male sexual partners has been recently disclosed as an independent predictor of clearance of cervical HR-HPV infections in women. ${ }^{2}$ In the HIM cohort, clearance of oncogenic HPV was more rapid 
in older males $(\mathrm{HR}=1.2$; 95\% CI: $1.01-$ 1.03). ${ }^{9}$

This cornerstone study has several important implications, some of which were addressed in the original report, and some others raised in the commentary of Dr Monsonego, ${ }^{10}$ which appeared in the same issue of the Lancet. Despite the many similarities (pinpointed above), this study also disclosed some important and yet unexplained differences in the natural history of genital HPV infections between women and males. One of those is the different risk profile across the age; while the risk of HPV decreases with increasing age in women, the present study failed to disclose the same to happen among the males. Indeed, men seem to have a stable risk for acquiring new HPV infections throughout their life, as shown in this study by the constant HPV incidence rates in men aged 18-70 years and residing in three different countries. ${ }^{9}$ Apart from the constant exposure to HPV (i.e., constant partner numbers across age groups), the authors speculated that these differences might have an immunological grounds. Due to the differences in the anatomic microenvironment, males have a lower prevalence of HPV antibodies than women, and for those who have circulating HPV antibodies, their titers are usually lower than in women. ${ }^{9}$ If these results can be confirmed, and if indeed, men remain at high risk of incident HPV infections even at older age, then HPV vaccination of older men might be warranted.

This brings us to the second potential implication of these HIM data, i.e., the feasibility of vaccinating males against HPV. ${ }^{11}$ This issue was raised in his commentary by Dr Monsonego, while discussing the currently available means of preventing HPV transmission from an infected male to his female partner. ${ }^{10}$ Given that the efficacy of condom use and circumcision in preventing HPV transmission still lacks evidence-based confirmation, it is easy to agree with his conclusions stating that ' $\mathrm{HPV}$ vaccination of men will protect not only them but will also have implications for their sexual partners'. ${ }^{10}$ Interestingly, this timely statement coincides with the first documented evidence that prophylactic vaccination of males with the quadrivalent HPV vaccine (Gardasil) is highly effective against penile and anal precancer lesions due to the vaccine HPV genotypes, published just a few weeks ago. ${ }^{11}$

1 Kulmala SM, Shabalova I, Petrovitchev N, Syrjänen K, Gyllenstein $U$ et al. Type-specific persistence of highrisk human papillomavirus infections in the New Independent States of the former Soviet Union Cohort Study. Cancer Epidemiol Biomarker Prev 2007; 16: 17-22.
2 Louvanto K, Syrjänen KJ, Grénman SE, Rintala MA, Syrjänen SM. Genotype-specific clearance of genital human papillomavirus (HPV) infections among mothers in the Finnish Family HPV Study. J Clin Microbiol 2010; 48: 2665-71.

3 Hippeläinen MI, Syrjänen S, Hippeläinen MJ, Koskela $\mathrm{R}$, Pulkkinen J et al. Prevalence and risk factors of genital human papillomavirus (HPV) infections in healthy males. A study on Finnish conscripts. Sex Transm Dis 1993; 20: 321-8.

4 Hippeläinen MI, Hippeläinen M, Saarikoski S, Syrjänen K. Clinical course and prognostic factors of human papillomavirus infections in men. Sex Transm Dis 1994; 21: 272-9.

5 DeCarvalho JJ, Syrjänen KJ, Jacobino M, Rosa NT, Carvalho LZ. Prevalence of genital human papillomavirus (HPV) infections established with different diagnostic techniques among males attending an urological clinic. Scand J Urol Nephrol 2006: 40: 138-43.

6 Elbasha EH, Dasbach EJ. Impact of vaccinating boys and men against HPV in the United States. Vaccine 2010; 28: 6858-67.

7 Kjaer SK, Munk C, Winther JF, Jorgensen HO, Meijer $\mathrm{CJ}$ et al. Acquisition and persistence of human papillomavirus infection in younger men: a prospective follow-up study among Danish soldiers. Cancer Epidemiol Biomarkers Prev 2005; 14 : 1528-33.

8 Partridge JM, Hughes JP, Feng Q, Winer RL, Weaver $\mathrm{BA}$ et al. Genital human papillomavirus infection in men: incidence and risk factors in a cohort of university students. J Infect Dis 2007; 196: 112836.

9 Giuliano AR, Lee JH, Fulp W, Villa LL, Lazcano E et al. Incidence and clearance of genital human papillomavirus infection in men (HIM): a cohort study. Lancet 2011; 377: 932-40.

10 Monsonego J. Genital infection with HPV in men: research into practice. Lancet 2011; 377: 881-3.

11 Giuliano AR, Palefsky JM, Goldstone S, Moreira ED Jr, Penny ME et al. Efficacy of quadrivalent HPV vaccine against HPV infection and disease in males. $N \mathrm{Eng} / \mathrm{J}$ Med 2011; 364: 401-11. 